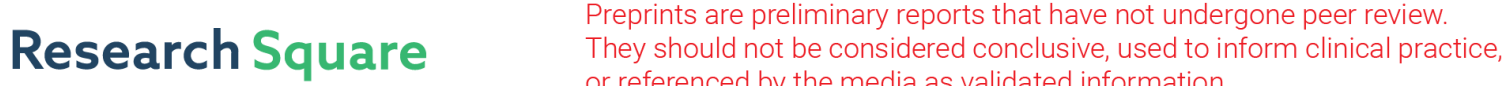 \\ or referenced by the media as validated information. \\ Elastomeric Seal Stress Analysis Using Photoelastic Experimental Hybrid Method
}

\section{Bruno Mose}

Jomo Kenyatta University of Agriculture and Technology

Dong-Kil Shin ( $\sim$ dkshin@yu.ac.kr)

Yeungnam University

\section{Bernard Alunda}

Taita Taveta University

Jeong-Hwan Nam

Dongyang University

\section{Research Article}

Keywords: Elastomeric seals, stress analysis, stress freezing, photoelasticity

Posted Date: October 26th, 2021

DOl: https://doi.org/10.21203/rs.3.rs-990522/v1

License: (c) (1) This work is licensed under a Creative Commons Attribution 4.0 International License. Read Full License 


\title{
Elastomeric seal stress analysis using photoelastic experimental hybrid
}

\section{Method}

\author{
${ }^{1}$ Bruno R. Mose, ${ }^{2}$ Dong-Kil Shin ${ }^{*},{ }^{3}$ Bernard O. Alunda, and ${ }^{4}$ Jeong Hwan Nam \\ ${ }^{I}$ School of Mechanical, Manufacturing and Materials Engineering, Jomo Kenyatta University of Agriculture \& \\ Technology, Kenya \\ ${ }^{2}$ School of Mechanical Engineering, Yeungnam University, 214-1, Dae-dong, Gyeongsan, Gyeongbuk 712-749, \\ Korea \\ ${ }^{3}$ School of Mines and Engineering, Taita Taveta University, Kenya \\ ${ }^{4}$ Department of Mechanical System Engineering, Dongyang University, 145 Dongyangdaero, Punggi Yeongju, \\ Gyeongbuk, 750-711, Korea
}

*Corresponding Author: E-mail address: dkshin@yu,ac.kr, Tel.: +82 538102457

ORCID: Bruno Robert Mose-0000-0002-3300-7917, Bernard Alunda- 0000-0003-0283-6525

\begin{abstract}
Stress freezing is an important and powerful procedure in 3-dimensional experimental stress analysis using photoelasticity. The application of the stress freezing technique to extract stress components from loaded engineering structures has, however, declined over the years even though its principles are well established. This is attributed to huge costs arising from energy consumption during the process. In addition, significant time is needed to generate the desired information from isoclinic and isochromatic fringes. To overcome the limitations of stress freezing in photoelasticity and transform it into an economical device for stress analysis in an engineering environment, a new stress freezing cycle that lasts 5 hours is proposed. The proposed technique is used in several applications of elastomeric seals with different cross-sectional profiles to assess its suitability. It was found that reducing the cycle time can lead to huge energy savings without compromising the quality of the fringes. Moreover, the use of isochromatic only to extract stress components leads to a shorter processing time to achieve desirable information since the process of obtaining isoclinic data is involving. In this paper, results of stress analysis from stress frozen elastomeric seals with various cross-sections using the new stress freezing cycle are presented.
\end{abstract}

Keywords: Elastomeric seals, stress analysis, stress freezing, photoelasticity 


\subsection{Introduction}

When certain polymeric materials with birefringence capabilities are heated above the glass transition temperature and loaded, then slowly cooled to normal room temperature, the stresses induced by the loads will remain intact even after the load is removed [1-2]. The model specimen made from such polymeric materials with optically sensitive properties can be cut into slices and studied to determine the stress state in the component. This forms the basis of the stress freezing phenomenon, which has been widely used in 3-dimensional photoelasticity for the experimental analysis of stresses in complex structures. This technique has been recognized as an important and powerful method to extract stress components from loaded engineering structures. However, its usage has declined over the years due to huge costs arising from energy consumption during the process. Moreover, significant time is required to generate the desired data from isoclinic and isochromatic fringes.

Drucker [3] had predicted the "imminent death" of photoelastic experimental stress computations and their place taken by computer-based techniques such as finite element method FEM [4]. The computer-based tools for stress analysis were introduced with a lot of excitement capable of allowing researchers to verify without the need of developing prototypes. Also, they enable simultaneous calculation and visualization of dynamic physical phenomena in addition to the extrapolation of experimental results. However, the accuracy of such tools has come into question especially considering the numerous assumptions made in the formulation of the solutions. Additionally, most of the computational software is very complex and expensive making it unavailable in the hands of most scientists.

Experimental stress analysts have in recent years rediscovered experimental methods as being more realistic tools and this has led to a re-birth of 3-dimensional photoelasticity [5]. Modern manufacturing technologies such as rapid prototyping and digital photoelasticity have also made 3-D photoelasticity an attractive alternative tool for stress analysis compared to FEM. It has been used for example, in the validation of 
designs with complicated geometries. Although 3-dimensional photoelasticity is steadily being incorporated into the engineering design process, certain challenges need to be overcome.

Over the years, elastomeric seals with O-shaped profiles commonly known as O-rings, have found wide use for sealing applications to control fluid flow under high pressure from escaping from one compartment to the other in pumps, boilers, nuclear power plants, heat exchangers, etc [6]. The O-rings are reported to offer a practical method to the ultimate hydraulic seal. During installation, however, the O-ring may twist. Consequently, the elastomeric O-ring assumes a corkscrew shape which is commonly known as a torsional failure as indicated in Fig. 1. Other limitations of the O-ring include the low performance at high temperatures and high rubbing speeds. Disregarding this limitation may result in poor performance of the seal. However, in circumstances where the design specifications allow, the use of O-rings seals ensures lasting and dependable service.

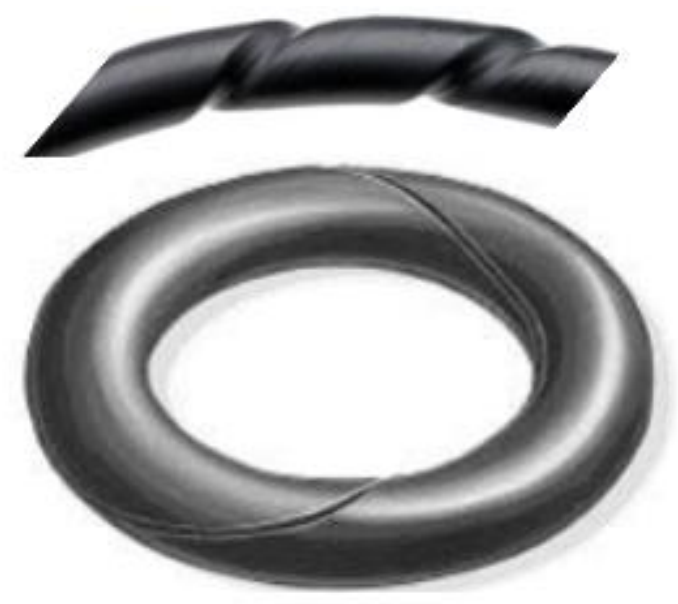

Fig. 1. Torsional failure of O-ring

Various cross-sectional profiles have been proposed to minimize torsional failure commonly observed in O-rings Fig 2 (a). Some of the profiles that offer alternative and better sealing capabilities are the D-ring, X-ring, and square ring (Fig. 2 b-d). The D-ring seal, for instance, has a large and flat geometric base that stops it from twisting and rolling during installation and operation, preventing torsional failure. D-ring seals 
also offer the advantage that they can be manufactured to standard sizes to replace the O-rings. The X-ring on the other hand has 4 lobes specially designed to improve the lubrication of the seal and prevention of the rolling effect common in O-ring seals. Moreover, the X-rings allow operation in a wide pressure and temperature range and high reciprocating speeds.

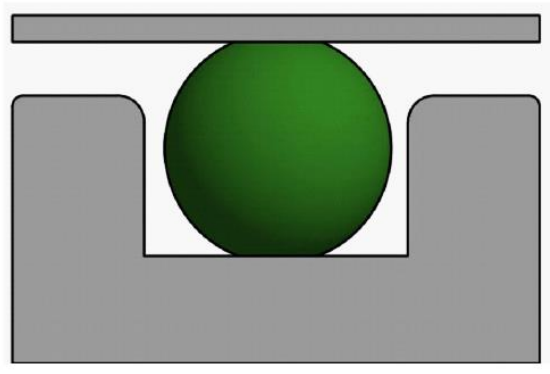

(a)

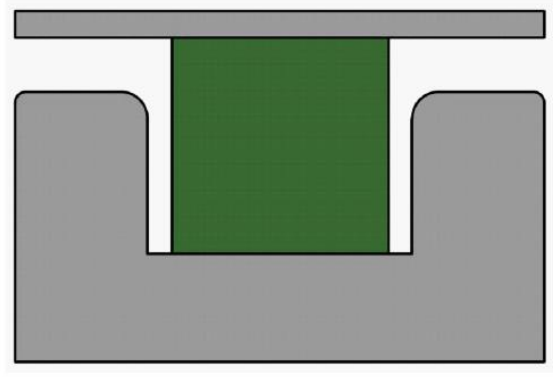

(c)

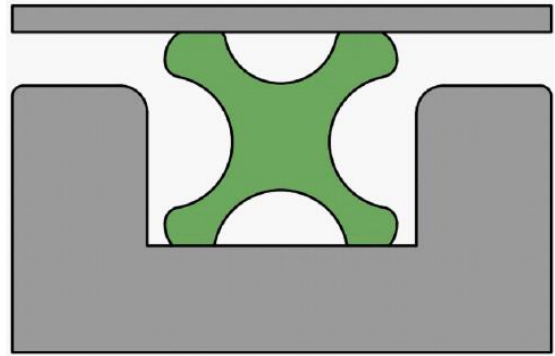

(b)

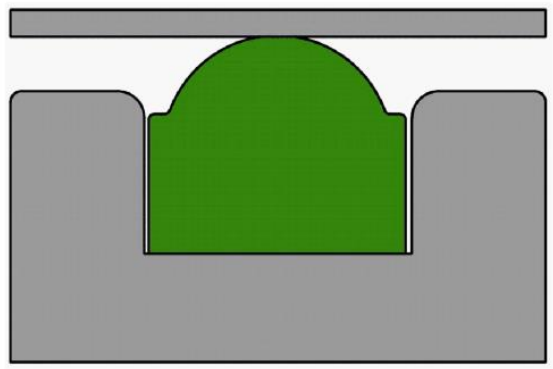

(d)

Fig. 2. Elastomeric seals with various cross-sectional profiles; (a) O-ring (b) X-ring (c) square ring (d) D-ring.

In this paper, new stress freezing cycle for photoelastic models of complex geometries is proposed with the advantage of huge savings on energy requirements compared to previously utilized cycles [7-11]. The proposed cycle is applied in stress freezing of elastomeric seals made from an epoxy made from Araldite (B41) and cured with Hardener (HT 903) with different cross-sectional profiles to assess its suitability. The cross-sectional profiles considered include the D-ring, square ring, and X-ring. These profiles were selected over the O-ring due to its inherent limitations. 


\subsection{Theoretical Background}

\subsection{Hertz Contact Theory}

In a plane problem, the stress components can be easily represented using Muskhelishvilli's potential functions $\xi$ and $\vartheta$ in conjunction with the Airy stress function as shown in Equation (1) [12]. It is to be noted that the stress components comprise of two (2) complex functions $\xi(\mathrm{z})$ and $\vartheta(\mathrm{z})$ which if determined then the stress components can be obtained.

$$
\begin{aligned}
& \sigma_{x}=\operatorname{Re}\left[2 \xi(\mathrm{z})-\bar{z} \xi^{\prime}(z)-\vartheta(\mathrm{z})\right] \\
& \sigma_{y}=\operatorname{Re}\left[2 \xi(\mathrm{z})-\bar{z} \xi^{\prime}(z)+\vartheta(\mathrm{z})\right] \\
& \tau_{x y}=\operatorname{Im}\left[\bar{z} \xi^{\prime}(z)+\vartheta(\mathrm{z})\right]
\end{aligned}
$$

From theory, it is assumed that stress functions $\xi(\mathrm{z})$ and $\vartheta(\mathrm{z})$ are analytic functions, and can be represented in terms of power series, as shown in equation (2).

$$
\begin{aligned}
& \xi(\mathrm{z})=\sum_{n=0}^{N} P_{n} z^{\frac{n}{2}} \\
& \vartheta(\mathrm{z})=\sum_{n=0}^{N} Q_{n} z^{\frac{n}{2}}
\end{aligned}
$$

It can be shown that the relative equation between the complex coefficients of the complex function is as shown in equation (3).

$$
Q_{n}=-\frac{n}{2} P_{n}-\overline{P_{n}}
$$

Substituting the relative equation between the complex coefficients of the complex function into the stress functions and replacing the resulting stress functions into Equation (1), equation (4) is determined 


$$
\begin{aligned}
& \sigma_{x}(z)=\sum_{n=1}^{N} \operatorname{Re}\left\{P_{n}[2 R(n, z)-S(n, z)]+\overline{P_{n}} R(n, z)\right\} \\
& \sigma_{y}(z)=\sum_{n=1}^{N} \operatorname{Re}\left\{P_{n}[2 R(n, z)+S(n, z)]+\overline{P_{n}} R(n, z)\right\} \\
& \tau_{x y}=\sum_{n=1}^{N} \operatorname{Im}\left\{P_{n} S(n, z)-\overline{P_{n}} R(n, z)\right\}
\end{aligned}
$$

Where $R(n, z)=\frac{n}{2} z^{\frac{n}{2}-1}$ and $S(n, z)=\frac{n}{2}\left[\left(\frac{n}{2}-1\right) \bar{z}-\frac{n}{2} z\right] z^{\frac{n}{2}-2}$

The beauty of using photoelasticity is that it can quantify stresses throughout a 3D structure and determine the stress gradients however, it requires birefringent material. The maximum and minimum principal stresses are expressed using Equations (5.a) and (5.b) respectively.

$$
\begin{aligned}
& \sigma_{1}=\frac{\sigma_{x}+\sigma_{y}}{2}+\sqrt{\left(\frac{\sigma_{x}-\sigma_{y}}{2}\right)^{2}+\tau_{x y}^{2}} \\
& \sigma_{2}=\frac{\sigma_{x}+\sigma_{y}}{2}-\sqrt{\left(\frac{\sigma_{x}-\sigma_{y}}{2}\right)^{2}+\tau_{x y}^{2}}
\end{aligned}
$$

\subsection{Stress analysis by Photoelastic Experimental Hybrid Method}

To extract the stress components in a structure under various loading conditions using photoelastic data, it is important to develop mathematical relations between stresses, sample specimens, and the optical parameters such as refraction indices, a fringe constant used in photoelasticity. For an isotropic material, Equation (6) is used to describe the stress optic law [13].

$$
\sigma_{1}-\sigma_{2}=\frac{N_{f} f_{\sigma}}{t}
$$

By combining Equations (5.a), (5.b), and 6, Equation 7 can be easily obtained.

$$
\left(f_{\sigma}^{2} x N_{f}^{2}\right) t^{-2}-\left(\sigma_{x}-\sigma_{y}\right)^{2}-4 \tau_{x y}^{2}
$$

where $f_{\sigma}$ is the fringe value of the stress, $N_{f}$ is the fringe order, and $t$ is the specimen thickness. 
By combining the experimental and the analytical results, an error is always generated which is always a number other than zero. Therefore, a suitable numerical tool can be employed to determine and reduce the errors as shown in Equation 8.

From the photoelastic experiment, we can get useful data that can be processed to compute the stresses in loaded components. If the experimental data is substituted into equation (7), some errors, $D(\varepsilon)$, may arise as shown in equation (8). Therefore, $W(\varepsilon)$ is always a number other than zero. Efforts to minimize the errors include the use of numerical techniques [14]. When the errors reach the limiting condition of $W(\varepsilon) \leq 10^{-5}$, stable solutions are determined.

$$
\left(f_{\sigma}^{2} x N_{f}^{2}\right) t^{-2}-\left(\sigma_{x}-\sigma_{y}\right)^{2}-4 \tau_{x y}^{2}=W(\varepsilon)
$$

If Equation (4) is substituted into Equation (8), then equation (9) is obtained.

$$
\begin{aligned}
(\varepsilon)=\left(\frac{f_{\sigma} N_{f}}{t}\right)^{2} & -\left\{\sum_{n=1}^{N} a_{n} \operatorname{Re}[2 R(n, z)-2 S(n, z)]\right. \\
& \left.+\sum_{n=1}^{N} b_{n} \operatorname{Im}[2 R(n, z)+2 S(n, z)]\right\}^{2} \\
& -\left\{\sum_{n=1}^{N} a_{n} \operatorname{Im}[2 S(n, z)-2 R(n, z)]\right. \\
& \left.+\sum_{n=1}^{N} b_{n} \operatorname{Re}[2 R(n, z)+2 S(n, z)]\right\}^{2}
\end{aligned}
$$

When the thickness of the specimen $(t)$, the measured fringe orders $\left(N_{f}\right)$, the position coordinates of the measured fringe orders $(z=x+i y)$, and the value of the stress fringe $\left(f_{\sigma}\right)$ are substituted in Equation (9), the equation will have $a_{n}$ and $b_{n}$ as the only functions. When the experimental data and the Hook-Jeeves numerical method are applied to equation (9), $a_{n}$ and $b_{n}$ are easily determined when an acceptable error 
limit is achieved. The stress functions $\xi(\mathrm{z})$ and $\vartheta(\mathrm{z})$ can then be evaluated by plugging in the values of $a_{n}$ and $b_{n}$ in the respective equation. When the determined $\xi(\mathrm{z})$ and $\vartheta(\mathrm{z})$ are substituted into Equation (1), then it is possible to obtain the stress components $\sigma_{X}, \sigma_{Y}$ and $\tau_{X Y}$ that is developed in a given member when an arbitrary load is applied to it. The prescribed procedures are what comprise the photoelastic experimental hybrid method.

\subsection{Experiment and Experimental Method}

\subsection{Fabrication of photoelastic models}

The casting of the photoelastic models of the elastomeric seals with the cross-sectional profiles under investigation was done using Araldite (B41, Ciba-Geigy) and hardener (HT 903, Ciba-Geigy) at the ratio of 10:3 following the procedure developed earlier by Nam et al. [7]. The epoxy resin casting cycle is shown in Fig. 3. The epoxy resin was introduced by Leven [15] as a photoelastic material and they offer great impetus to photoelastic stress analysis compared to other resins such as phenolics resins, styrene alkyd, etc due to numerous advantages. The epoxy resins come closest to satisfying the requirements of ideal photoelastic materials owing to their ease of machinability, linear stress/fringe relation, shorter curing time, lack of residual stresses. The epoxy resin is also easy to cast into large sizes, transparent, highly resistant to creep, and has both mechanical and optical isotropy. The Araldite resin has been confirmed to be the convenient material for making birefringent models compared to the counterparts such as Flexible GIV, and PL2 [16]. 


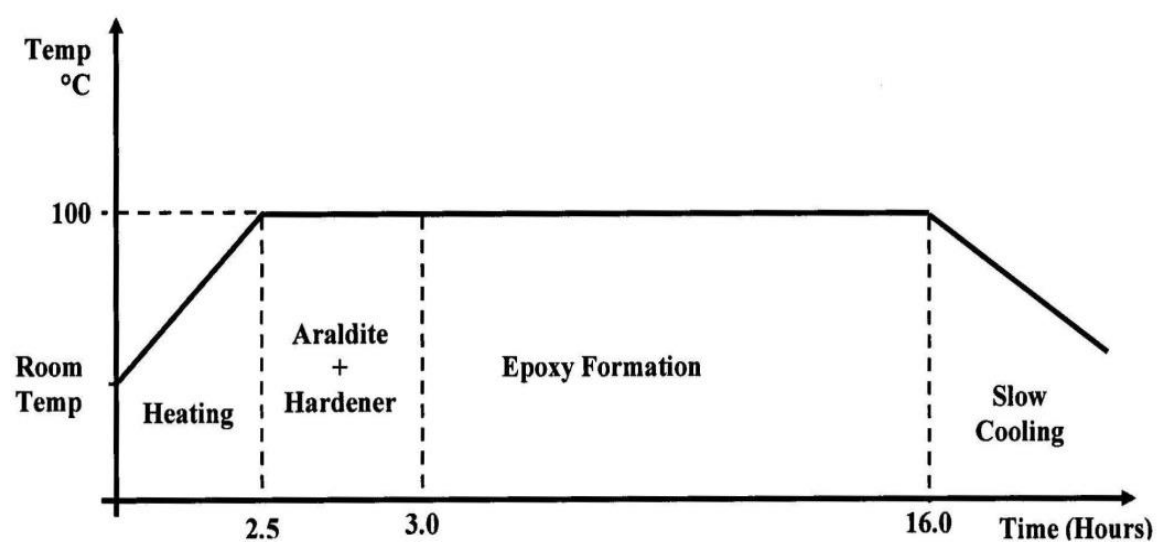

Fig. 3. Epoxy resin casting cycle.

\subsection{Stress Freezing cycle}

In a two-dimensional photoelastic experimental analysis $[17,18]$, a suitable model made from either epoxy or other materials is fabricated, loaded while it is inside the photoelastic experimental rig and the fringe pattern examined are photographed which are later interpreted. However, in a real situation especially in the industries, complicated problems exist which are 3-dimensional and so the stress analysis of such problems requires special techniques. One of the techniques employed is the stress freezing method which permits the construction of the desired model, loading, it appropriately and later analyzing the interior planes of the model using photoelasticity [19]. In the stress freezing method, the deformations of the model and the related optical responses are locked into the loaded 3-dimensional model. The model is then sliced, polished, and positioned on the loading device of a transparent photoelastic experimental device to acquire the interior stress information.

The pioneering work of the stress freezing method was an initiative of Oppel [20], Kuske [21], Hetenyi [22], and Frocht [23] in Germany way back in 1936 but its usage was limited especially in the industry because it was time-consuming and so expensive though it was such a powerful tool. In his research, Jan Cernosek [24] showed the competitive and cost-effectiveness of three-dimensional photoelasticity. 
The experimental apparatus mainly consists of the experimental equipment, specially designed to provide the seals with different geometries with a constant compression rate and varying internal pressure. The experimental equipment was designed with an opening at the top where the internal pressure can be applied using a hydraulic pump as shown in Fig. 4(a). In addition, the device has a valve to allow the opening and closing of the opening following the stress freezing cycle.

The old stress freezing cycle consisted of consistently heating the specimen to a temperature above the glass transition temperature of the epoxy (usually above $120^{\circ} \mathrm{C}$ ) before loading (application of the internal pressure), holding it there for approximately $120 \mathrm{mins}$ before cooling it at a rate of $5^{\circ} \mathrm{C} / \mathrm{hr}$ up to a temperature of about $90^{\circ} \mathrm{C}$ before switching off and allowing the whole assembly to cool inside the furnace to room temperature. The whole process for stress freezing would require the furnace to run for at least 12 hours.

Stress freezing of the various seals was installed in the photoelastic experimental rig and compressed with a uniform squeeze rate of $20 \%$ followed by the application of the various internal pressures. The experimentation of the seals with various cross-sectional profiles was carried out using the newly proposed stress freezing cycle and involved the following steps;

1. The seals fabricated from epoxy resin and the experimental equipment were placed in the furnace and separately heated for roughly one (1) hour at a constant temperature of $125^{\circ} \mathrm{C}$ (just above the end of the glass transition temperature, ) in the stress-freezing furnace so that the epoxy resin attains the glass transition temperature, $T_{g}$.

2. The seals (one at a time), and the experimental equipment were assembled inside the furnace to impact the required compression rate and the required internal pressure.

3. The assembled experimental equipment was heated at that temperature for 120 mins before being allowed to cool at a rate of $10^{\circ} \mathrm{C} / \mathrm{hr}$ up to the beginning of the glass transition temperature.

4. The assembled experimental equipment was permitted to cool to ambient temperature inside the furnace. 
The old and new stress freezing cycles were plotted in the same graph as shown in Fig. (4b) for comparison. The new freezing cycle required the furnace to operate for only about 5 hours.

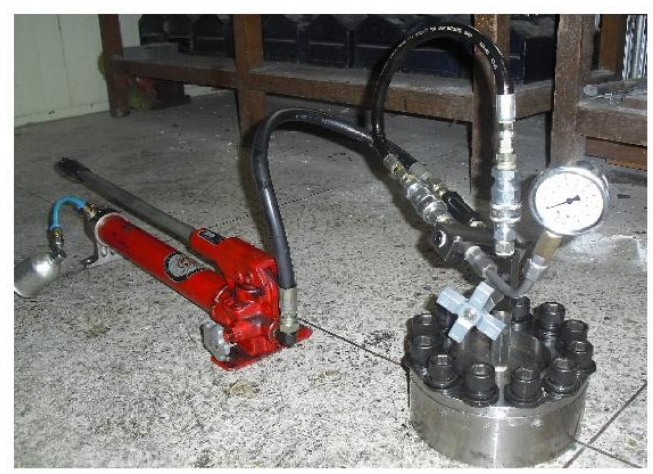

(a)

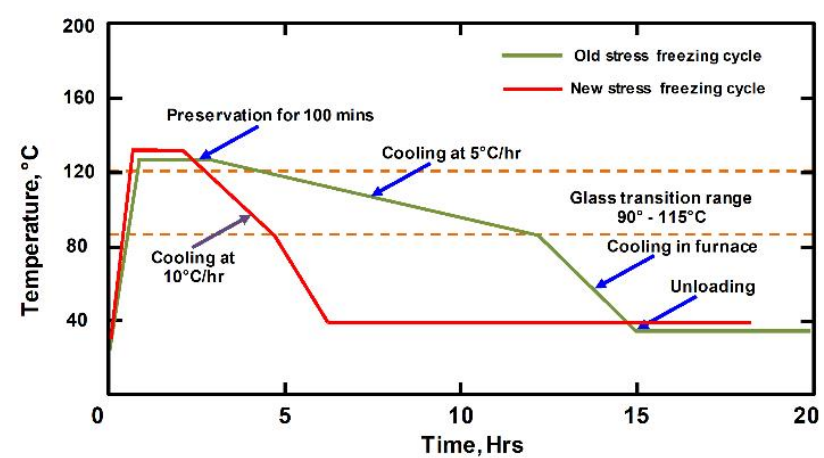

(b)

Fig.4. (a) Loading equipment capable of subjecting the seals to a uniform compression and internal pressure and (b) stress freezing cycles (old -Alunda et. al [25] and new proposed cycle.

After stress freezing, the seals with different geometries were sliced into thin sections for further analysis using the photoelastic experimental device shown in Fig. 5. The experimental device is composed of a light source for illumination followed by the polarizer which is a quarter-wave plate (No. 2), that is positioned amid the specimen (No.3) and the analyzer (No. 5), which is the $2^{\text {nd }}$ quarter-wave plate. The $2^{\text {nd }}$ quarterwave plate is the specimen and the high-speed camera (No. 6). Usually, the two (2) quarter-wave plates are added to achieve circularly polarized light that is capable of only producing the isochromatic fringes alone and not the isoclinic fringes. This allows for easy discrimination of the isoclinic and the isochromatic fringe patterns. The thin slices for analysis were cut using a saw and polished properly to a minimum thickness of about $0.8 \mathrm{~mm}$. To achieve good quality digital images, the polished slices were put in a box of glass-filled a mixed solution of organic alcohol and paraffin before positioning it on the transparent photoelastic experimental rig. A high-speed digital CCD camera (No. 6) was then used to capture the isochromatic fringe patterns then moved to a personal computer for further analysis. 


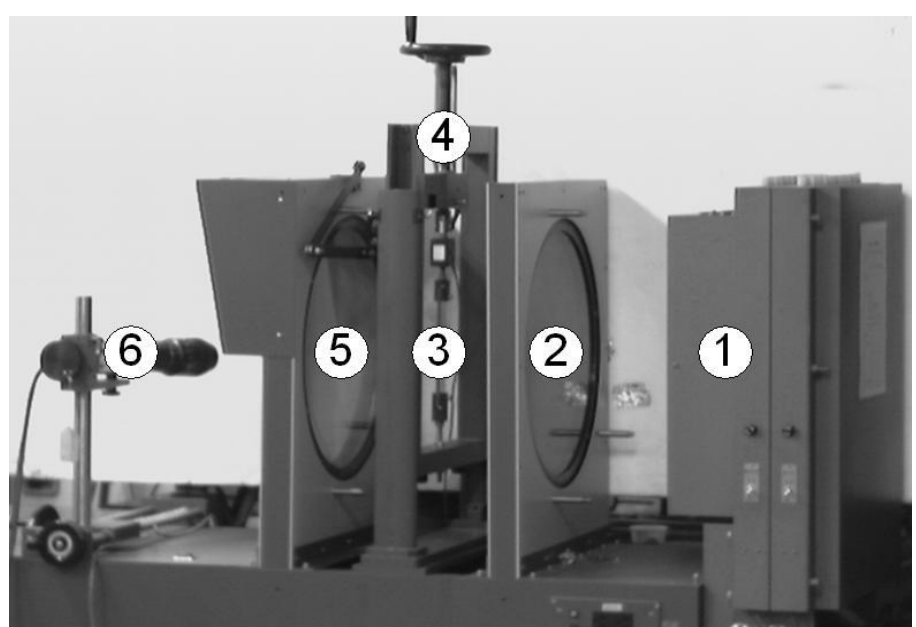

(1) - Light source, (2) - polarizer, (3) - specimen, (4) - loading device, (5) - analyzer (6) - high-speed camera.

Fig. 5. Schematic of transparent photoelastic experiment device.

\subsection{Experimental Results and Discussion}

The effectiveness of the proposed stress freezing cycle to yield good quality isochromatic fringes for further analysis was evaluated. It can be seen from Fig 6 that the cycle resulted in good quality isochromatic fringes which are very clear. The isochromatic fringe patterns are distinguishable and increase in numbers as one approaches the contact zones 
(I)

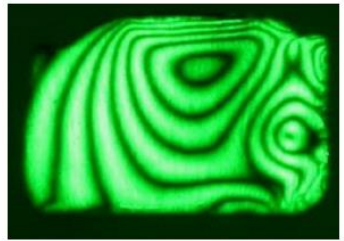

(a)

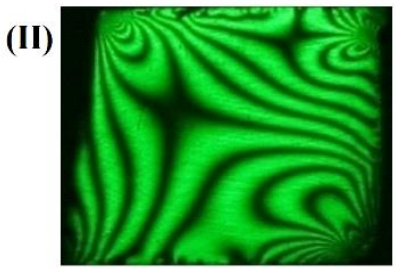

(a)

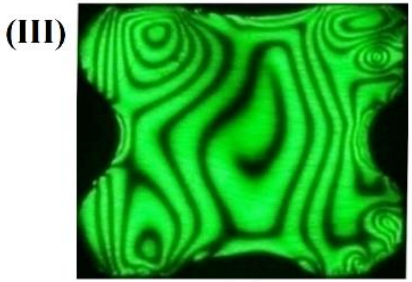

(a)

(a) $\mathrm{P}_{\mathrm{i}}=1.0 \mathrm{MPa}$

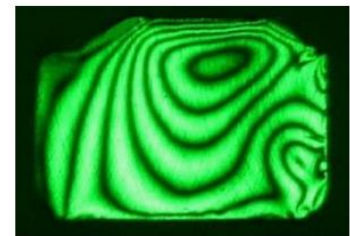

(b)

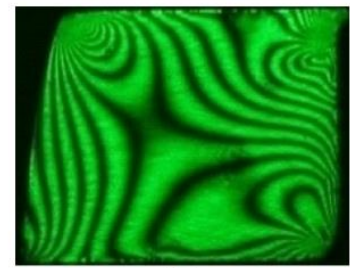

(b)

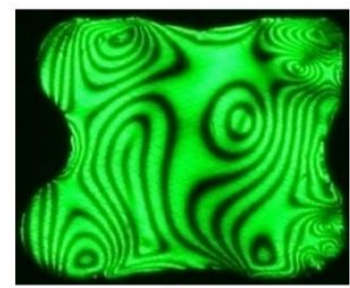

(b)

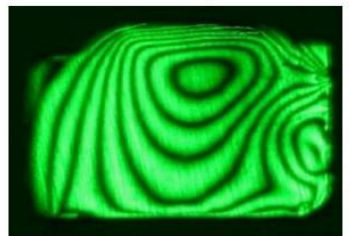

(c)

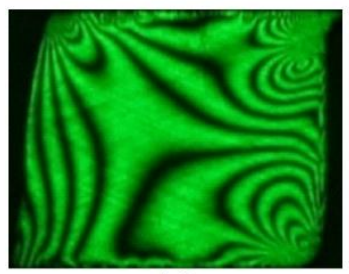

(c)

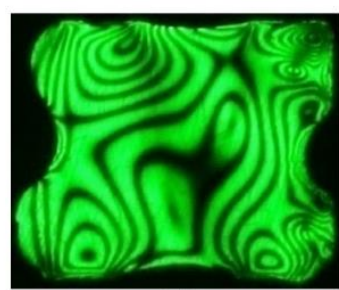

(c)

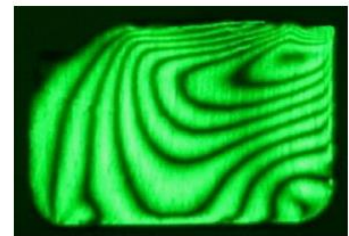

(d)

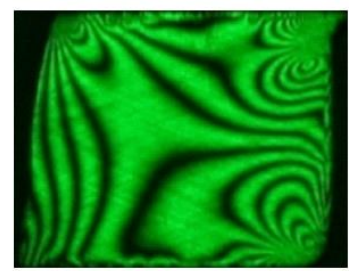

(d)

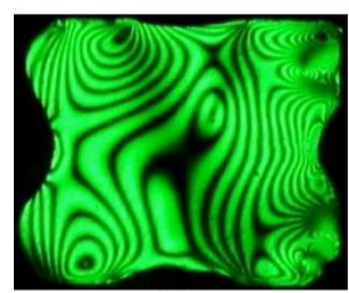

(d)

(b) $\mathrm{P}_{\mathrm{i}}=2.0 \mathrm{MPa}$

(c) $\mathrm{P}_{\mathrm{i}}=3.0 \mathrm{MPa}$

(d) $\mathrm{P}_{\mathrm{i}}=4.0 \mathrm{MPa}$

Fig. 6. The isochromatic fringe patterns of (I) O-ring, (II) square ring, and (III) X-ring were obtained using the photoelastic experimental rig at different internal pressures.

A representative analysis of the contact stresses was done for each seal geometry to ascertain the distribution of stresses on the upper surface under a uniform compression of $20 \%$ and internal pressure of $1.0 \mathrm{MPa}$. The photoelastic experimental hybrid method was employed in the analysis of the stresses of the contact problem. The required data from the experiment for the photoelastic experimental hybrid was obtained from the dotted quadrilateral box ( $\square$ ) in the actual isochromatic fringe pattern. The "+" marks used in the actual isochromatic show the positions from which the experimental data was gathered. The cross marks "+" used are assumed to be located at the center of each of the white or dark bands with little permitted errors. The indistinguishability of the actual and graphic isochromatic as shown in Fig. 7 suggests that the results obtained are true. Thus it can be deduced that the contact and interior stresses thus obtained are matching those of the actual specimen under consideration. 
(I)

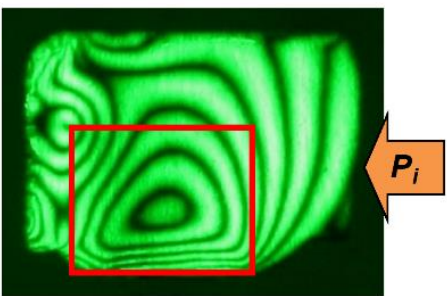

(a)

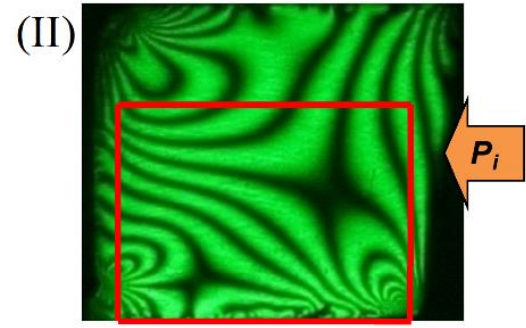

(a)

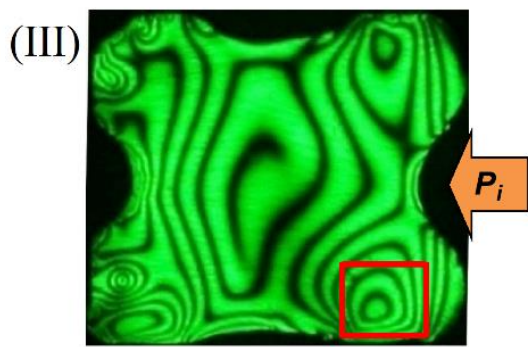

(a)

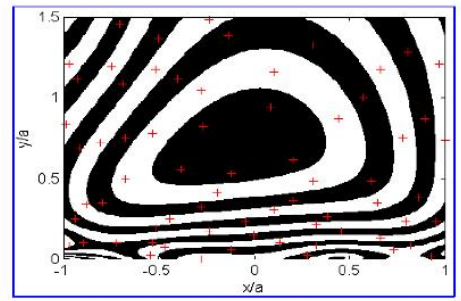

(b)

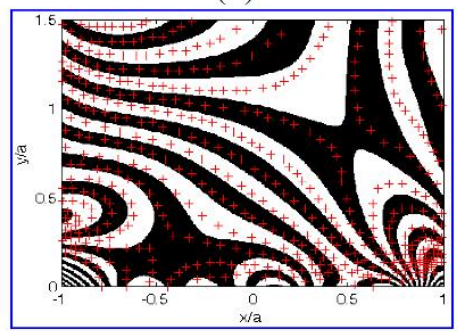

(b)

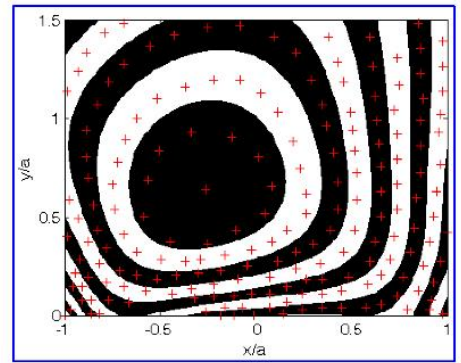

(b)

Fig. 7. The actual (a), and the graphical (b) isochromatic fringe patterns on the upper side of; (I) D-ring, (II) Square ring, and (III) X-ring from the photoelastic experimental hybrid method under a uniform compression of $20 \%$ and internal pressure $\left(P_{i}=1 \mathrm{MPa}\right)$.

The internal stress contours of the respective seal geometries were also generated as shown in Fig. 8. $P_{i}$ denotes the internal pressure used to normalize the internal stresses and the contact stresses. For the D-ring and square-rings, the normalized $\sigma_{X}, \sigma_{Y}$, and $\tau_{X Y}$ internal stresses were 3.3 $\mathrm{MPa}, 3.4 \mathrm{MPa}, 0.13 \mathrm{MPa}$, and 3.12 $\mathrm{MPa}$, 4.2 $\mathrm{MPa}$, and 0.68 $\mathrm{MPa}$ respectively. Also, the highest magnitudes of the normalized internal stresses $\sigma_{X}, \sigma_{Y}$, and $\tau_{X Y}$ for the X-ring were examined and found to be $3.25 \mathrm{MPa}, 3.25 \mathrm{MPa}$, and $0.125 \mathrm{MPa}$ respectively. A summary of the highest internal stresses for the three seal geometries is summarized in the bar graph Fig. 9. The high magnitudes of shear stress computed from the square ring indicates that it is more likely to fail compared to the X-ring and D-ring. 
(I)

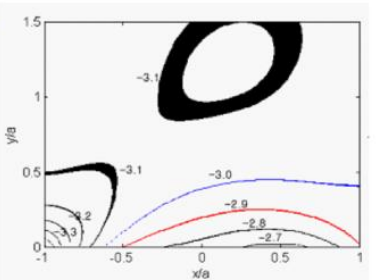

(a)

(II)

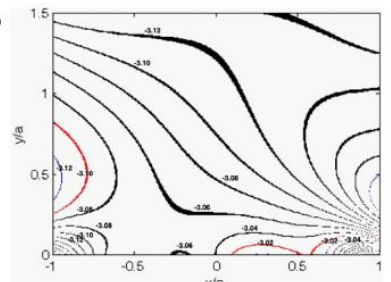

(a)

(III)

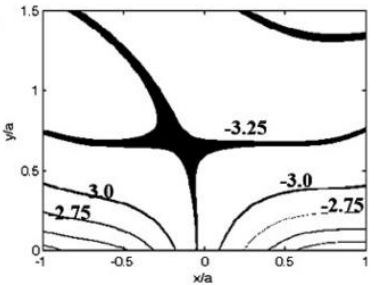

(a)

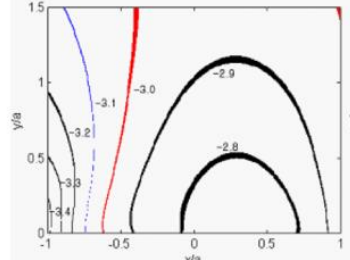

(b)

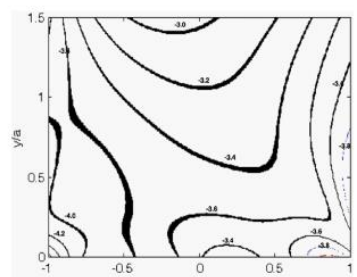

(b)

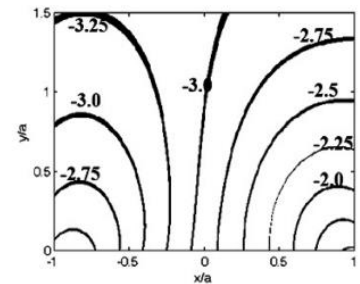

(b)

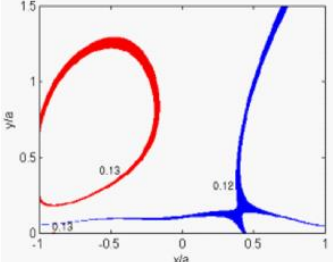

(c)

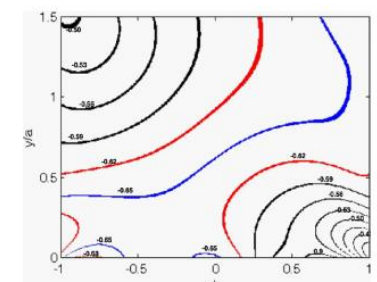

(c)

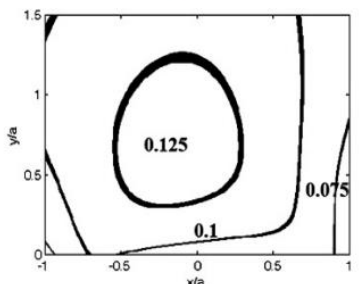

(c)

Fig. 8. The internal stress contours on the upper region of; (I) D-ring, (II) Square ring, and (III) X-ring under uniform compression of $20 \%$ normalized by internal pressure $\left(P_{i}\right)$ of $1 \mathrm{MPa}$. (a), (b), and (c) represents $\sigma_{X} / P_{i}, \sigma_{Y} / P_{i}$, and $\tau_{X Y} / P_{i}$ respectively.

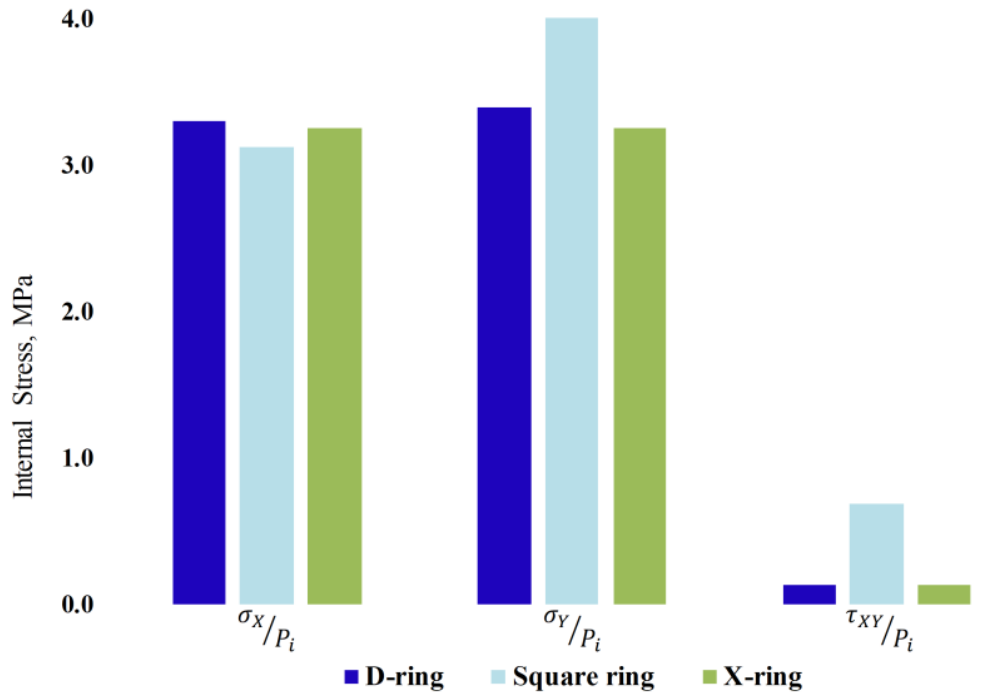

Fig. 9. A comparison of the performance of various seals with pressure applied. 


\subsection{Conclusion}

In this study, a novel stress freezing cycle that lasts 5 hours instead of 15 hours was proposed and its effectiveness was investigated using the photoelastic experimental hybrid method. It was found that;

1. The proposed stress freezing cycle can yield good quality isochromatic fringes for experimental stress analysis.

2. The huge costs arising from energy consumption can be saved by the use of the proposed stress freezing cycle

3. The square ring experienced the highest shear stress compared to the D-ring and X-ring. This means that the square ring is more likely to fail since shear stress is considered in engineering stress models as the most damaging as it controls crack initiation

4. Process of extraction of stress components $\sigma_{X}, \sigma_{Y}$, and $\tau_{X Y}$ from isochromatic fringes provides a more realistic tool for stress analysis

\section{Acknowledgments}

This research was supported by Basic Science Research Program through the National Research Foundation of Korea (NRF) funded by the Ministry of Education (NRF-2018R1D1A1A09083672)

\section{Disclosure statement}

The authors wish to state that there is no potential conflict of interest

\section{References}

1. D. Swain, J. Philip, S.A. Pillai, K. Ramesh "A Revisit to the Frozen Stress Phenomena in Photoelasticity," Experimental Mechanics, 56:903-917 (2016). 
2. Cernosek, J. Three-dimensional photoelasticity by stress freezing. Experimental Mechanics 20, 417-426 (1980).

3. Drucker, D.C., "Thoughts on the Present and Future Interrelation of Theoretical and Experimental Mechanics, " Experimental Mechanics, 8 (3), 97-106 (1968).

4. De Vree, J. H. P., Peters, M. C. R. B., \& Plasschaert, A. J. M., A comparison of photoelastic and finite element stress analysis in restored tooth structures. Journal of oral rehabilitation, 10(6), 505517 (1983).

5. Vieira, F. G., Scari, A. S., Magalhães Júnior, P. A. A., Martins, J. S., \& Magalhães, C. A. Analysis of Stresses in a Tapered Roller Bearing Using Three-Dimensional Photoelasticity and Stereolithography. Materials, 12(20), 3427 (2019).

6. Momon, S.; Garcia, J.; Issard, H. Leak tightness of O-rings for transport of radioactive material. Packag. Transp. Storage Secur. Radioact. Mater., 24, 3-9 (2013).

7. Nam Jeong-Hwan, Hawong Jai-Sug, Han Song-Ling and Park Sung-Han, "Contact Stress of Oring under Uniform Squeeze Rate by Photoelastic Experimental Hybrid Method", Journal of Mechanical Science and Technology, 22, 2337-2349 (2008).

8. Nam Jeong-Hwan, Hawong Jai-Sug, Dong-Chul Shin and Bruno R.M, "A Study on the Behaviors and Stresses of O-ring under Uniform Squeeze Rate and Internal Pressure by Transparent Type photoelastic Experiment," Journal of Mechanical Science and Technology, 25, 2427-2438 (2011).

9. Bruno R. Mose, Jai Sug Hawong, Bernard O. Alunda, Lim Hyun Seok and Jeong Hwan Nam, "Evaluating the stresses of a stepped unrounded D-ring under uniform squeeze rate and internal pressure by photoelastic experimental hybrid method," Journal of Mechanical Science and Technology, 26 (8), 2603-2616 (2012).

10. Bruno Robert Mose, Jeong Hwan Nam, Lim Hyun Seok and Jai-Sug Hawong, "Internal stress analysis of a stepped rounded D-ring under a uniform squeeze rate and internal pressure using a photoelastic experimental hybrid method.” Journal of Mechanical Science and Technology, 27 (8), 2413-2423 (2013) 
11. Bruno Robert Mose, Jai-Sug Hawong, Dong-Kil Shin, Lim Hyun Seok, Dong-Chul Shin "Stress and Fracture Analysis of D-ring by Photoelastic Experimental Hybrid Method," Journal of Mechanical Science and Technology, 31 (8), 3657-3660 (2017)

12. N. I. Muskhelishvili, Some Basic Problems of Mathematical Theory of Elasticity, 4th Edition, P. Noordhoff Ltd., Groningen Netherlands, (1963).

13. R. C. Sampson, A Stress-optic law for Photoelastic Analysis of Orthotropic Composites, Experimental Mechanics, 10, 210-215 (1970).

14. M. S. Bazaraa and C. M. Shetty, "Nonlinear Programming Theory and Algorithms," John Wiley \& Sons Inc. U.S.A. (1979).

15. Leven, M.M., Epoxy Resins for Photoelastic Use, Photoelasticity Proceedings of the International Symposium, Chicago, 145 (Oct. 1961).

16. Martins, Adriana Vieira, Wellington Márcio dos Santos Rocha, Nelson Renato França Alves Silva, Rodrigo de Castro Albuquerque, Allyson Nogueira Moreira, Rodrigo Richard da Silveira, Cláudia Silami Magalhães, and Wellington Antonio Soares. Relevance of Resin for Photoelasticity. Materials Research 22 (2019).

17. Schiavon, J. A., Tsuha, C. D. H. C., \& Esquivel, E. R. Application of Two-Dimensional Photoelastic Analysis Study of Helical Anchors. In 1st International Geotechnical Symposium on Helical Foundations, 262-271 (2013).

18. Topbasi, B., Gunday, M., Bas, M., \& Turkmen, C. Two-dimensional photoelastic stress analysis of traumatized incisor. Braz Dent J, 12(2), 81-84 (2001).

19. Touahir, Kamel, Ali Bilek, Philippe Bocher, and Mustapha Beldi. "Experimental and numerical analysis of the fields of the frozen stresses in an epoxy sphere using the stress-freezing method and mechanical slicing." Journal of Materials and Engineering Structures 6, no. 2 (2019).

20. Opel, G., "Polarisationsopttsche Untersuchung Ramticher Spannungs - und Dehnungszustande. “Forsch. Ing. Wes., 7, 240 (1936) 
21. Kuske, A., "Das Kunstharz Phenoljormaldehyd in der Spannungsoptic”, Forsch. Ing. Wes., 9, 139 (1938)

22. Hetenyi, M., "The Fundamentals of Three-Dimensional Photoelasticity”, Journal of Appl. Mech., A, 149 (1938).

23. Frocht, M.M., "Photoelasticity”, Vol. I1, John Wiley and Sons, New York (1948).

24. Jan Cernosek, Three-dimensional Photoelasticity by Stress Freezing, Symposium on Photomechanics in Engineering, Mason (1979)

25. B. O. Alunda, D. O. Agumba, N. A. Mutua, L. O. Otieno, I. O. Ondicho, J.S. Hawong, Investigating the Performance of Square Ring under Uniform Squeeze Rate and Internal Pressure, Mat.-wiss. u. Werkstofftech, 51, 1653-1661 (2020). 